\title{
Gulf War Illness and Inflammation: Association of symptom severity with C-reactive protein
}

\author{
Lisa M. James 1,2,3, Brian E. Engdahl',2,4, Rachel A. Johnson', Apostolos P. Georgopoulos 1,2,3,4,5* \\ 'Brain Sciences Center, Department of Veterans Affairs Health Care System, Minneapolis, MN, 5541, USA \\ ${ }^{2}$ Department of Neuroscience, University of Minnesota Medical School, Minneapolis, MN 55455, USA \\ ${ }^{3}$ Department of Psychiatry, University of Minnesota Medical School, Minneapolis, MN 55455, USA \\ ${ }^{4}$ Department of Psychology, University of Minnesota Medical School, Minneapolis, MN 55455, USA \\ ${ }^{5}$ Department of Neurology, University of Minnesota Medical School, Minneapolis, MN 55455, USA
}

Article Info

\section{Article Notes}

Received: February 28, 2019

Accepted: April 10, 2019

\section{${ }^{*}$ Correspondence:}

Dr. Apostolos P. Georgopoulos, Brain Sciences Center (11B),

Minneapolis VAHCS, One Veterans Drive, Minneapolis, MN 55417, USA; Email: omega@umn.edu.

(C) 2019 Georgopoulos AP. This article is distributed under the terms of the Creative Commons Attribution 4.0 International License

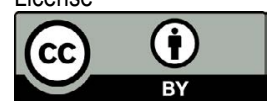

Keywords:

Gulf War Illness

Inflammation

C-reactive protein

Neuroimmune

Persistent Antigens

Immunity
Abstract

Gulf War Illness (GWI) is a chronic multi-system condition that has affected one-third of U.S. veterans who served in the Persian Gulf. Although GWI etiology remains unclear, mounting evidence points to immune system involvement and inflammation, in particular, as underlying the host of symptoms associated with the condition. Here we investigated the association between GWI symptoms and C-reactive protein (CRP), a marker of inflammation, in 76 veterans with GWI. Results indicated a highly significant positive association between CRP and mean GWI symptom severity. At the symptom domain level, CRP was significantly and positively associated with Pain, Neurocognitive/Mood, Fatigue, and Respiratory symptom severity but not with Skin or Gastrointestinal symptom severity. These results support the premise that GWI symptoms, particularly those implicating brain involvement, are a result of neuroinflammation. The cause for inflammation is not known. We have hypothesized that at the root of GWI are harmful persistent antigens stemming from environmental exposures associated with service during the Gulf War that could not be successfully eliminated due to lack of specific immunity ${ }^{1,2}$. Work is underway in our laboratory to identify and eliminate persistent antigens in veterans with GWI which we anticipate will result in reduced inflammation and reduced GWI symptoms.

\section{Introduction}

Gulf War Illness is a chronic disease of unclear etiology that has affected a large number of veterans of the 1990-1991 Persian Gulf War. Symptoms affect several systems and include fatigue, musculoskeletal pain, neurological and cognitive impairment, and mood disruptions ${ }^{3}$ in addition to respiratory, gastrointestinal, and dermatological complaints ${ }^{4}$. Burgeoning evidence suggests that genetic vulnerability related to immune system functioning coupled with environmental insults may underlie the host of symptoms observed in $\mathrm{GWI}^{1,2}$.

Increasingly, immune system disruption has been recognized in relation to $\mathrm{GWI}^{1,5-14}$. Consistent with evidence of immune-mediated loss of blood brain barrier integrity ${ }^{15}$, alterations in brain structure and function have been associated with $\mathrm{GWI}^{16-19}$. Furthermore, brain function in GWI has been shown to be indistinguishable from that of known immune-related conditions ${ }^{20}$. Robust evidence of immune involvement in conjunction with brain alterations suggests GWI is best characterized as a neuroimmune condition ${ }^{7,20}$ resulting from persistent antigens that contribute to immune system disruption and inflammation ${ }^{2}$.

Evidence of inflammation has been reported in veterans with $\mathrm{GWI}^{10,21,22}$. For example, C-reactive protein (CRP), a non-specific acute- 
phase biomarker of inflammation, has been shown to be elevated in $\mathrm{GWI}^{10,21}$. In fact, of 61 plasma proteins evaluated in one study of GWI ${ }^{10}, \mathrm{CRP}$ was one of only 6 that significantly differed between veterans with and without GWI and the only protein identified from a stepwise multivariate logistic regression model that contributed to a diagnostic model of GWI (along with lymphocytes, and monocytes). CRP is synthesized primarily in the liver hepatocytes; however, other cell types including smooth muscle cells, macrophages, endothelial cells, lymphocytes, and adipocytes have also been shown to synthesize $\mathrm{CRP}^{23}$. Notably, emerging evidence also indicates local CRP production in human neuronal cells of patients with Alzheimer's disease and upregulation of CRP in Alzheimer's-affected brain areas ${ }^{24}$. Finally, CRP has historically been viewed as a marker of inflammation that arises in response to inflammatory cytokines such as interleukin 6; yet, mounting evidence suggests CRP may also play a causal role in inflammation ${ }^{23}$. Thus, CRP may both signal and potentiate inflammation in various cell types and tissues, perhaps underlying the diffuse symptoms involving multiple systems as seen in GWI. To date, however, relatively little is known about CRP as it relates to GWI. Here we aim to evaluate the association between GWI symptoms and CRP levels to further assess the link between CRP and GWI symptomatology.

\section{Materials and Methods}

Participants: A total of 76 veterans with GWI and no comorbidities were studied (70 men, age $56.3 \pm 8.1$ y [mean $\pm \mathrm{SD}$ ], 6 women, age $50.5 \pm 5.2 \mathrm{y}$ ). GWI status was determined using a self-report symptom checklist that evaluates the presence and severity of various symptoms comprising 6 domains characteristic of GWI: fatigue, pain, neurological/mood/cognitive, gastrointestinal, skin rashes, and respiratory. Items were rated on a scale from 0 (absent) to 3 (severe). Veterans who met either Center for Disease Control ${ }^{1}$ or Kansas criteria ${ }^{2}$ for GWI were included in the present analyses. All study protocols were approved by the appropriate Institutional Review Boards. Study participants provided informed consent, in adherence to the Declaration of Helsinki, and were financially compensated for their time.

CRP: Non-fasting peripheral venous blood samples were collected for evaluation of high sensitivity $C$ reactive protein and analyzed using standard procedures by the Minneapolis VAHCS Clinical Laboratory.

Other variables: The Body Mass Index (BMI) was 31.65 \pm 5.21 (mean $\pm \mathrm{SD}, \mathrm{N}=76$ ). No participant reported illegal drug use or alcohol abuse. Twenty-one participants were receiving medications (15 on antidepressants, 4 on pain relievers, 2 on beta blockers); five were receiving opioids for pain relief ( 3 tramadol, 2 hydrocodone).
Data analysis: The IBM-SPSS statistical package (version 23) was used to analyze the data. The average GWI symptom severity across all domains was computed as well as the average symptom severity within each of the 6 GWI symptom domains. The correspondence between GWI symptoms and CRP were evaluated using stepwise linear regressions., where GWI symptom severity was the dependent variable, CRP was the independent variable, and medication status and BMI were covariates.

\section{Results}

CRP. CRP values were distributed in a non-normal fashion (Figures 1 and 2). Therefore, they were transformed to their logarithms to normalize their distribution (Figures 3 and 4). The log-transformed CRP values, $C R P^{\prime}$, were used in all subsequent analyses:

$$
C R P^{\prime}=\ln (C R P)
$$

Association of GWI symptom severity with CRP. The mean of GWI symptom severity (across the $6 \mathrm{GWI}$ symptom

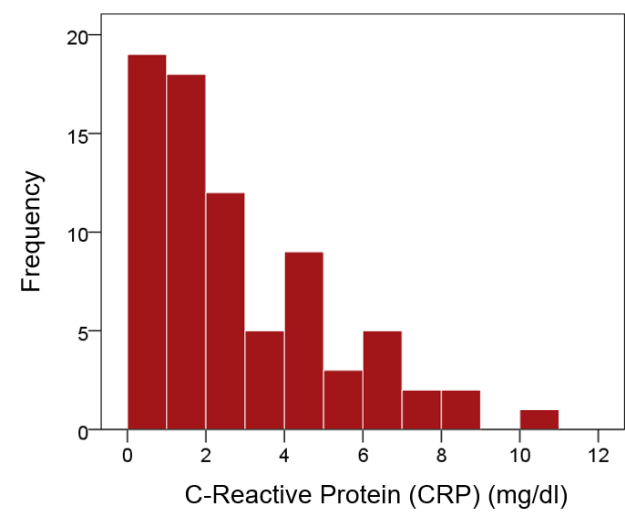

Figure 1: Exponential distribution of CRP values.

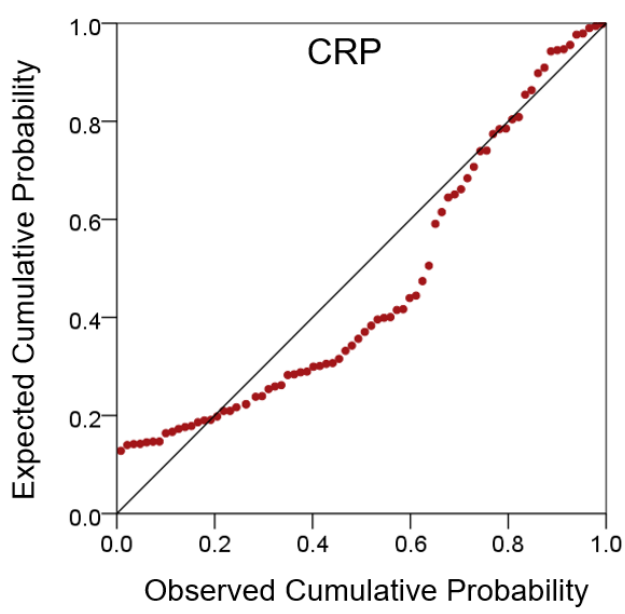

Figure 2: Probability plot of CRP values, under the assumption of a normal distribution. A substantial deviation from normality is indicated by the deviation of the plotted values from the midline. 
domains) was significantly and positively associated with $\ln (\mathrm{CRP})\left[C R P^{\prime}\right.$, equation 1] (Figure 5; $\mathrm{r}=0.353, \mathrm{P}=0.002$ ). The results of the stepwise regression analyses are shown in Table 1.

It can be seen that $C R P^{\prime}$ had a significant effect in all but the Gastrointestinal and Skin domains. BMI did not have a significant effect in any analysis, whereas medication status had a significant effect in all but the Respiratory domain.

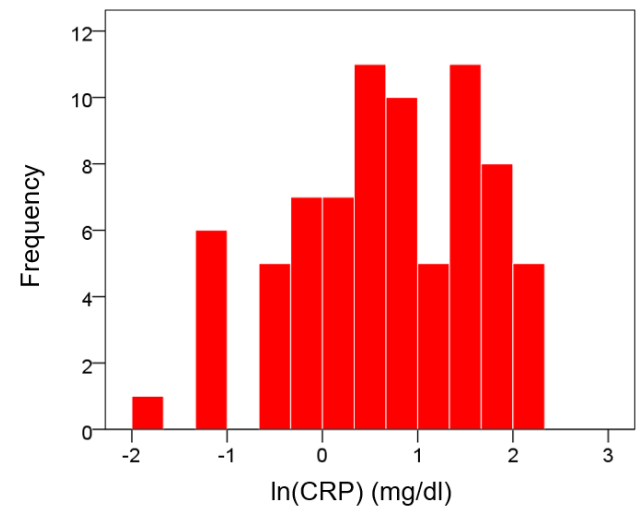

Figure 3: Log-transformed distribution of CRP values.

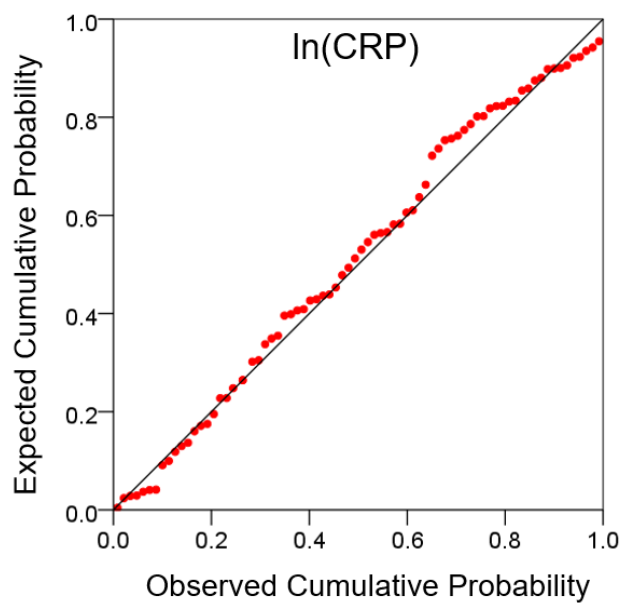

Figure 4: Probability plot of log-transformed CRP values, under the assumption of normal distribution. Notice the closeness of the plotted values to the midline.

\section{Discussion}

Here we investigated the association between inflammation and GWI symptoms in a sample of GW veterans and found a highly significant positive association between CRP, a marker of inflammation, and GWI symptom severity. The results add to the literature highlighting the role of inflammation in $\mathrm{GWI}^{10}$ and point to the potential benefit of interventions for GWI aimed at reducing inflammation.

GWI is a chronic condition characterized by widespread symptoms spanning several systems including the central nervous system, respiratory, dermatologic, and gastrointestinal system. Of these, the brain is prominently involved with three of the six symptom domains - fatigue, pain, and neurocognitive/mood symptoms - implicating brain involvement. Notably, all three of these domains were highly significantly associated with CRP in the present study, further cementing GWI as a neuroimmune condition ${ }^{20}$. Respiratory symptoms were the only other domain that was significantly associated with inflammation.

Based on a series of recent findings in our lab we have proposed that GWI is a result of persistent antigens stemming from environmental exposures associated with service during the Gulf War that could not be successfully eliminated due to lack of specific immunity ${ }^{25}$. Initial support

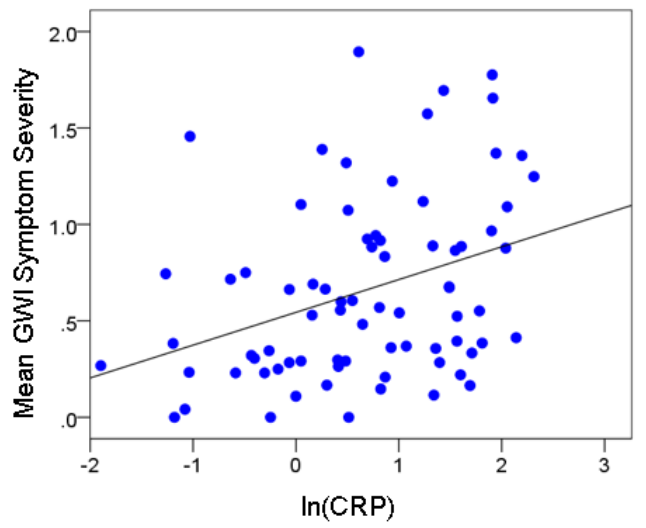

Figure 5: Association of mean GWI symptom severity with logtransformed CRP.

Table 1. Result of the stepwise regression analyses. P-values are those obtained for the final-step model; "excluded" denotes absence of the variable from this model.

\begin{tabular}{|l|c|c|c|}
\hline \multicolumn{1}{|c|}{ Dependent variable (symptom score) } & Independent variable & \multicolumn{2}{c|}{ Covariates } \\
\hline GWI symptom domain & $C R P^{\prime}$ & Medication status & $\mathrm{BMI}$ \\
\hline Mean symptom severity & $\mathrm{P}<0.001$ & $\mathrm{P}<0.001$ & $\mathrm{P}=0.242$ (excluded) \\
\hline Fatigue & $\mathrm{P}<0.001$ & $\mathrm{P}<0.001$ & $\mathrm{P}=0.838$ (excluded) \\
\hline Pain & $\mathrm{P}=0.013$ & $\mathrm{P}=0.024$ & $\mathrm{P}=0.163$ (excluded) \\
\hline Neurocognitive & $\mathrm{P}=0.004$ & $\mathrm{P}<0.001$ & $\mathrm{P}=0.977$ (excluded) \\
\hline Respiratory & $\mathrm{P}=0.018$ & $\mathrm{P}=0.317$ (excluded) & $\mathrm{P}=0.618$ (excluded) \\
\hline Gastrointestinal & $\mathrm{P}=0.097$ (excluded) & $\mathrm{P}=0.012$ & $\mathrm{P}=0.112$ (excluded) \\
\hline Skin & $\mathrm{P}=0.603$ (excluded) & $\mathrm{P}=0.045$ & $\mathrm{P}=0.323$ (excluded) \\
\hline
\end{tabular}


of the "Persistent Antigen" hypothesis was established on findings demonstrating that 6 Class II human leukocyte antigens (HLA) distinguish healthy Gulf War veterans from veterans with GWI ${ }^{1}$. Specifically, the 6 alleles were significantly more common among healthy veterans suggesting that their presence is protective against GWI; conversely, the absence of these HLA alleles and consequent lack of protection results in GWI. Subsequently, we demonstrated that one of the 6 protective alleles, in particular - HLADRB1*13:02 - is highly protective against brain atrophy ${ }^{2,26}$ that has been observed in GWI veterans ${ }^{16}$. The protection provided by the presence of these class II alleles is inherent in their function which is elimination of foreign antigens via antibody production; however, the ability to stimulate antibody production hinges on a match between HLA proteins and epitopes derived from foreign antigens. In the absence of a match, the antigen persists, resulting in inflammation (reflected here by elevated CRP) and other detrimental effects including cell damage and atrophy. Thus, we suspect that prominent GWI symptoms, particularly those implicating brain involvement, are a result of neuroinflammation due to the persistence of foreign antigens resulting from lack of HLA protection.

Although the specific cause of inflammation in GWI veterans remains to be elucidated, we hypothesize that it is the result of the existence of harmful persistent antigens in GWI; indeed, we have studies underway aimed at identifying persistent antigens in veterans with GWI with the goal of ultimately eliminating them (and thereby reducing inflammation) via personalized immunotherapy. Two recent in vitro studies in our lab have provided initial evidence supporting immunotherapy as a promising intervention for GWI. Specifically, we have demonstrated that serum from veterans with GWI results in detrimental changes to cell morphology in neural cultures; however, the addition of serum from healthy Gulf War veterans ${ }^{27}$ or human immunoglobulin $\mathrm{G}(\operatorname{IgG})^{28}$ neutralize those damaging effects. The neutralizing effects are presumed to result from the ability of antibodies present in serum from healthy Gulf War veterans and in pooled IgG to eliminate persistent antigens in veterans with GWI. We anticipate that elimination of persistent antigens would result in reduced inflammation and reduced GWI symptoms. Inflammatory response regulation via monoclonal antibodies targeting specific cytokines or neuroendocrine control of the cytokine network, as is under investigation in other diseases ${ }^{29}$ may prove to be useful alternative therapeutic strategies for reducing GWI-related inflammation and symptoms.

\section{Acknowledgements}

This work was partially supported by the Department of Defense (Award Number W81XWH-15-1-0520), University of Minnesota (the American Legion Brain Sciences Chair) and the U.S. Department of Veterans Affairs. The sponsors had no role in the current study design, analysis or interpretation, or in the writing of this paper. The contents do not represent the views of the U.S. Department of Veterans Affairs or the United States Government.

\section{Author Contributions}

Contributed to data collection: LMJ, RAJ. Contributed to participant recruitment and evaluation: BEE, LMJ, RAJ. Contributed to study design: APG, BEE, LMJ. Contributed to data analysis: APG, LMJ. Wrote the paper: LMJ, APG. Contributed to editing the paper: All.

\section{References}

1. Georgopoulos AP, James LM, Mahan MY, et al. Reduced Human Leukocyte Antigen (HLA) protection in Gulf War Illness (GWI). EBioMedicine. 2016; 3: 79-85

2. James LM, Christova P, Engdahl BE, et al. Human leukocyte antigen (HLA) and Gulf War Illness (GWI): HLA-DRB1*13:02 spares subcortical atrophy in Gulf War veterans. EBioMedicine. 2017; 26: 126-131.

3. Fukuda K, Nisenbaum R, Stewart G, et al. Chronic multisymptom illness affecting Air Force veterans of the Gulf War. JAMA. 1998; 280: 981-988.

4. Steele L. Prevalence and patterns of Gulf War illness in Kansas veterans: association of symptoms with characteristics of person, place, and time of military service. Am J Epidemiol. 2000; 152: 992-1002.

5. Broderick G, Ben-Hamo R, Vashishtha S, et al. Altered immune pathway activity under exercise challenge in Gulf War Illness: an exploratory analysis. Brain Behav Immun. 2013; 28: 159-169.

6. Broderick G, Fletcher MA, Gallagher et al. Exploring the diagnostic potential of immune biomarker coexpression in Gulf War Illness. Methods Mol Biol. 2012; 934: 145-164.

7. Coughlin SS. A neuroimmune model of Gulf War Illness. J Environ Health Sci. 2017; 3: 106.

8. Hotopf M, David A, Hull L, et al. Role of vaccinations as risk factors for ill health in veterans of the Gulf war: cross-sectional study. BMJ. 2000; 320: 1363-1367.

9. Israeli E. Gulf War Syndrome as a part of the autoimmune (autoinflammatory) syndrome induced by adjuvant (ASIA). Lupus. 2012; 21: 190-194.

10. Johnson GJ, Slater BC, Leis LA, et al. Blood biomarkers of chronic inflammation in Gulf War Illness. PLoS One. 2016; 11(6): e0157855

11. Moss JI. Gulf War illnesses are autoimmune illnesses caused by increased activity of the p38/MAPK pathway in CD4+ immune system cells, which was caused by nerve agent prophylaxis and adrenergic load. Med. Hypotheses. 2013; 81: 1002-1003

12. Parkitny L, Middleton S, Baker K, et al. Evidence for abnormal cytokine expression in Gulf War Illness: A preliminary analysis of daily immune monitoring data. BMC Immunol. 2015; 16: 57.

13. Skowera A, Hotopf M, Sawicka E, et al. Cellular immune activation in Gulf War veterans. J Clin Immunol. 2004; 24: 66-73.

14. Whistler T, Fletcher MA, Lonergan W, et al. Impaired immune function in Gulf War Illness. BMC Med Genomics. 2009; 2: 12 (8794-2-12)

15. Esposito P, Gheorghe D, Kandere K, et al. Acute stress increases permeability of the blood-brain-barrier through activation of brain mast cells. Brain Res. 2001; 888: 117-127

16. Christova P, James LM, Engdahl BE, et al. Subcortical brain atrophy in Gulf War Illness. Exp Brain Res. 2017; 235: 2777-2786. 
17. James LM, Engdahl BE, Leuthold AC, et al. Brain correlates of human leukocyte antigen (HLA) protection in Gulf War Illness (GWI) EbioMedicine. 2016; 13: 72-79.

18. Engdahl BE, James LM, Miller RD, et al. A magnetoencephalographic (MEG) study of Gulf War Illness (GWI). EBioMedicine. 2016 Oct 1; 12 127-32.

19. White RF, Steele L, O'Callaghan JP, et al. Recent research on Gulf War illness and other health problems in veterans of the $1991 \mathrm{Gulf}$ War: effects of toxicant exposures during deployment. Cortex. 2016; 74: 449-475.

20. Georgopoulos AP, James LM, Carpenter AF, et al. Gulf War illness (GWI) as a neuroimmune disease. Exp Brain Res. 2017; 235(10): 3217-3225

21. Johnson GJ, Leis LA, Slater BC, et al. Elevated platelet count, C-reactive protein and thromboxane analog-induced platelet aggregation in patients with Gulf War veterans' illnesses: evidence of a chronic inflammatory state? Blood Coagul. Fibrinolysis. 2013; 24: 736-741.

22. Kelsall HL, McKenzie DP, Sim MR, et al. Physical, psychological, and functional comorbidities of multisymptom illness in Australian male veterans of the 1991 Gulf War. Am J Epidemiol. 2009; 170: 1048-1056

23. Sproston NR, Ashworth JJ. Role of C-reactive protein at sites of inflammation and infection. Frontiers Immunol. 2018; 9.
24. Yasojima K, Schwab C, McGeer EG, et al. Human neurons generate C-reactive protein and amyloid P: upregulation in Alzheimer's disease. Brain Res. 2000; 887: 80-89.

25. James LM, Georgopoulos AP. Persistent antigen hypothesis: The human leukocyte antigen connection. J Neurol Neuromed, in press.

26. James LM, Christova P, Lewis SM, et al. Protective Effect of Human Leukocyte Antigen (HLA) Allele DRB1* 13: 02 on Age-Related Brain Gray Matter Volume Reduction in Healthy Women. EBioMedicine. 2018; 29: 31-7.

27. Georgopoulos AP, Tsilibary EP, Souto EP, et al. Adverse effects of Gulf War Illness (GWI) serum on neural cultures and their prevention by healthy serum. J Neurol Neuromed. 2018; 3(2): 19-27.

28. Tsilibary CEP, Souto EP, James LM, et al. Human immunoglobulin G (IgG) neutralizes adverse effects of Gulf War Illness (GWI) serum in Neural cultures: Paving the way to immunotherapy for GWI. J Neurol Neuromed. 2018; 3(5): 23-28.

29. Lissoni P, Messina G, Cenaj V, et al. The role of IL-17 secretion in mediating the influence of stress on cancner and other human systemic diseases. MOJ Immunol. 2018; 6(5): 180-183. 